\title{
Modeling Energy Costs and Emissions for Anycast RWA in Optical Data Center Networks
}

\author{
Arash Deylamsalehi, Pegah Afsharlar, and Vinod M. Vokkarane \\ Electrical and Computer Engineering Department, University of Massachusetts Lowell \\ Arash_Deylamsalehi@student.uml.edu, Pegah_Afsharlar@student.uml.edu, and Vinod_Vokkarane@uml.edu
}

\begin{abstract}
Energy consumption of network and data-center operators causes economic and environmental concerns. Geographical distribution of resources implies agreements with multiple utility companies; these are increasingly offering pricing and update the fuel diversity real-timely, in addition to standard flat-rate prices. We propose two algorithms to reduce operational expenditures and pollutions by choosing the route with the least cost and emissions for the consumed energy. Today data replicas are typically accessible from several optical data centers. Replicas lead to more flexible network routing. We propose an analytical modeling to calculate the electricity cost and emissions in the network with the number of replicas. The greatest benefit of our approach is that the network can dynamically adapt to renewable resources generation sudden rises or drops; high emissions are avoided ${ }^{1}$.
\end{abstract}

Index Terms-Real-time Pricing; Anycast; Green Networking; Routing and Wavelength Assignment.

\section{INTRODUCTION}

With the rise of cloud computing and storage, big data, and bandwidth-hungry applications, the number and size of data centers have increased exponentially. Big companies have been investigating billions of dollars to build new data centers and upgrade old ones, to provide cloud-based service to their customers. Moreover, the high bandwidth available due to deployed technologies makes the opportunity to provide new kinds of services by cloud data centers.

The number and placement of data centers have a strong impact on the service efficiency of data center networks (DCNs) [1]. Adding more replicas improves reliability and lowers latency across the network, but also increases the total network costs. Data center management systems prefer to operate networks in a partially replicated condition. Partially replicated means that objects might be stored at more than one data center, but not at all of the available data centers [2]. In this paper, we assume that a request has access to the entire object at single location. This model was defined based on the sharding practice. In this practice, the object is partitioned, and each piece is replicated at several locations, and is called shard [3]. A shard is accessible when a request assigns the available wavelength between the user and any replica location. Google's gmail service uses two replicas ${ }^{2}$, and Microsoft replicate every action in three data centers [4]. The authors of [4] find that with proper coding, a relatively small number of

\footnotetext{
${ }^{1}$ This work has been supported by the NSF CARGONET project under grant CNS-1406370.

${ }^{2}$ http://googleforwork.blogspot.com/2010/03/disaster-recovery-bygoogle.html
}

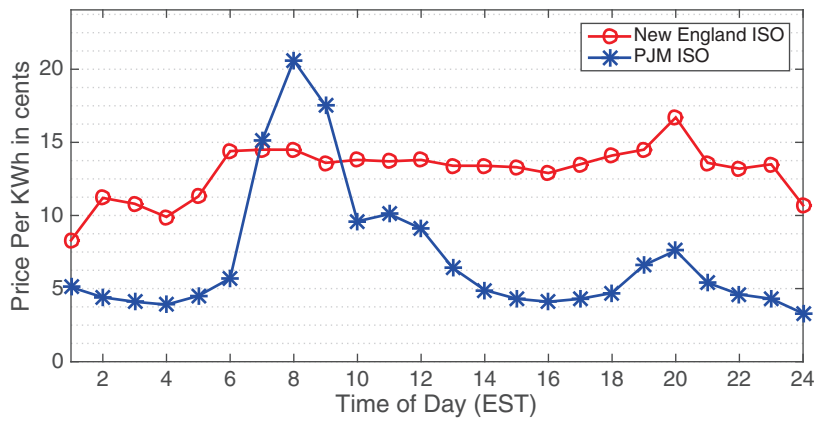

Fig. 1: Real-time hourly energy prices for February 20, 2015 in NEISO and PJM ISO power markets

two or three replicas is sufficient to provide data to customers reliably.

We can define such demand routing as an anycast request in order to sufficiently reach one of the data centers. Anycasting refers to a method of routing data from a source node to any node in a group of potential destinations [5]. In this paper, we consider the routing and wavelength assignment problem (RWA), in which each connection is set up along a physical network route and a single fixed wavelength there upon [6]. The authors of [7] present an anycast routing and wavelength assignment algorithm (ARWA) for WDM networks. An effective scheduling approach for anycast in optical Grids is presented in [8].

Data centers and communication networks around the world consume about 500 to 750 TWh combined annually [9], which has been increasing with growing the cloud computing. It causes environmental and economic concerns about the future of DCN. By considering $\$ 60 / \mathrm{MWh}$ as electricity cost and $526.6 \mathrm{gCO} 2 \mathrm{e} / \mathrm{kWh}$ for emissions, it is estimated that network communications and data centers are charged 30 to 45 billon dollars, and pollute 263 to 394 Mega ton emissions every year. Thus, even a small improvement in energy management can save hundreds of million dollars of electricity cost and reduce millions of tons in emissions. A large contribution to the energy consumption is the result of storing the data in the data centers, however in the reverse direction, getting data from data centers. Due to the geographical extension of wide area networks, which may be large as a continent, they are served by multiple utility companies. Figure 1 compares the electricity price of $\mathrm{NEISO}^{3}$ at Boston, MA and $\mathrm{PJM} \mathrm{ISO}^{4}$ at

\footnotetext{
${ }^{3}$ http://www.iso-ne.com/isoexpress/

${ }^{4}$ https://rrtp.comed.com/live-prices/?date $=20140713$
} 
Chicago, IL. Renewable generations of utility companies are fluctuating during the day, by changing the direct sunlight or wind speed. Additionally, if the network spans different timezones, there are guaranteed different peak-hours for both network traffic and power grid load. Considering the fuel diversity and electricity price of utility companies and replicated data storage architecture, we propose two algorithms to find the most cost effective and emissions effective data source and network route.

The rest of this paper is organized as follows: Section II discusses the electricity market models that form the basis of our approach. Section III contains the nodal and network-wide power consumption models. In Section IV we describe the implementation of our proposed Least Dollar Path and Least Emissions Path approach for distributed replica applications. Then we present our analytical models in Section V. We discuss the results of simulating our approach on a realistic network in Section VI and conclude the paper in Section VII.

\section{Electricity Market Model}

Electricity is generated in power plants by using the various origin resources; e.g. the United States fleet has a fuel diversity of coal (39\%), natural gas (27\%), nuclear (19\%), Hydro (6\%), and other renewable resources (6\%) in 2014 [10], then the generated electricity transmits through the transmission and distribution lines to consumers. Due to lack of economic technologies for storing the electricity, there is no way to store the large amount of electricity. Thus, the electricity grid is governed by the principle of unit commitment: generated electricity must be equal to demand. If enough energy is not generated, blackouts occur. Excess generation results in instabilities in voltage and frequency. Due to the essentiality of this equilibrium in the systems, independent system operators (ISO) are responsible to keep the system reliable, cleared and balanced in electricity markets. There are seven main electricity markets in the United States (refer to Fig. 2).

Each power distribution company supplies number of power consumers. power distribution companies are responsible to buy their required electricities from the power plants by bidding in the electricity markets, and sell it to their consumers by various rate models. Due to the unit commitment principle, the wholesale electricity prices fluctuate day-to-day and also throughout a given day [11]. Power distribution companies take these fluctuations into account by charging the real-time pricing (RTP), or charge their costumer flat rate by adding the margin to over the average electricity cost. For residential customers for example, one of the first areas where RTP became available was the Chicago area market. Studies have found that without the change of behavior, an average household customer has saved $1 \%$ or $2 \%$ over the duration of a year employing this service model [11].

Because of the lack of economic technologies for storing the electricity, there is no way to store the large amount of clean energy for high demand hours. Thus, fuel diversity differs from the power market to power market and time to time, depending the cost and availability of origin resources. Fuel

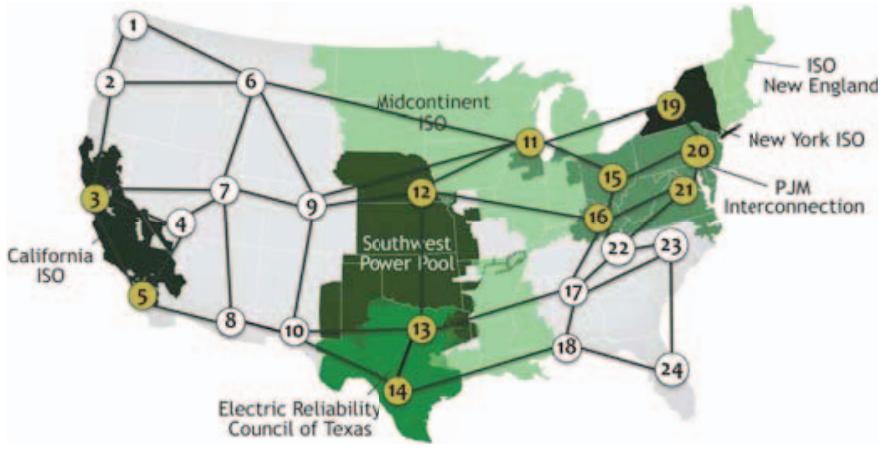

Fig. 2: 24-node USnet Topology.

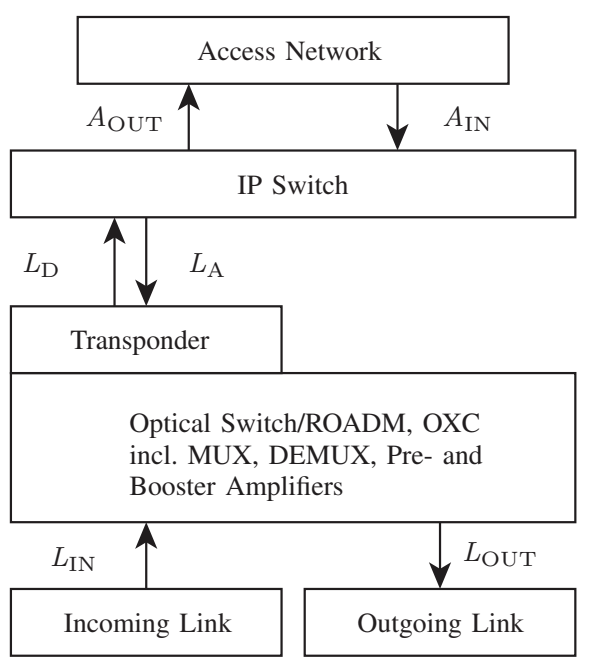

Fig. 3: Multilayer node power model.

diversity presents how the generated electricity break down to multiple generation methods which emit different amounts of emissions, e.g. coal (800 to $1050 \mathrm{gCO} e / \mathrm{kWh}$ ), natural gas (430 gCO2e/kWh), hydro (6 gCO2e/kWh), etc. The CO2e uses as emissions unit, and the "e" means all values include the impact of all kind of emissions. Based on fuel diversity and variety in the polluted air per $\mathrm{kWh}$, the emissions factor of electricity markets represents total produced emissions in scale of gCO2e by consuming $1 \mathrm{kWh}$ in the electricity markets. Due to fluctuation of fuel diversity, the emissions factor is various through a day.

\section{Power Nodal Model}

There are different models of network equipment power consumption in literature. We use a model that is based on real equipment measurements and accounts for both idling power consumption as soon as a node is turned on, and bandwidth usage dependency. In Fig. 3 we illustrate the multilayer (optical, electronic, and IP layer) node model used to calculate the network power consumption. We use values from [12] where a comprehensive analysis of equipment from different manufacturers resulted in the quantities listed in Tables I and II. The node consists of an electrical routing layer at the top, an optical transport and multiplexing layer at the bottom, and a connecting Optical Electronic Optical (OEO) layer in between. 
TABLE I

Network-Specific Variables

\begin{tabular}{l|c|c|c} 
Symbol & Description & Value & Unit \\
\hline$c$ & Transponder transmission rate & 10 & $G b p s$ \\
$\pi_{\mathrm{TX}}(c)$ & Transponder power consumption & 0.05 & $k W$ \\
$\pi_{\mathrm{OXC}}$ & OXC power consumption & 0.1 & $k W$ \\
$\pi_{\mathrm{IP}}$ & IP layer power consumption & 0.01 & $k W / G b p s$ \\
\hline
\end{tabular}

TABLE II

Node-Specific Variables

\begin{tabular}{l|c|c}
\hline Symbol & Description & Unit \\
\hline$A_{\text {IN }}$ & Input traffic from access network & $G b p s$ \\
$A_{\text {OUT }}$ & Output traffic to access network & $G b p s$ \\
$L_{\mathrm{D}}$ & Lightpaths dropped from WDM to IP (O-E) & integer \\
$L_{\mathrm{A}}$ & Lightpaths added to WDM from IP (E-O) & integer \\
$L_{\mathrm{IN}}$ & Incoming lightpaths & integer \\
$L_{\mathrm{OUT}}$ & Outgoing lightpaths & integer \\
\hline
\end{tabular}

We use WDM as transport network technology, since it is widely employed in high-speed wide area networks.

$$
\begin{aligned}
& p_{\mathrm{IP}}=\pi_{\mathrm{IP}}\left(A_{\mathrm{IN}}+A_{\mathrm{OUT}}+c\left(L_{\mathrm{D}}+L_{\mathrm{A}}\right)\right) . \\
& p_{\mathrm{OEO}}=\pi_{T X}(c)\left(L_{\mathrm{D}}+L_{\mathrm{A}}\right) . \\
& p_{\mathrm{WDM}}=\pi_{O X C}\left(L_{\mathrm{D}}+L_{\mathrm{A}}\right)+\alpha\left(L_{\mathrm{IN}}+L_{\mathrm{OUT}}\right)+\beta . \\
& P_{j}=p_{\mathrm{IP}}+p_{\mathrm{OEO}}+p_{\mathrm{WDM}} .
\end{aligned}
$$

At the source of data the layers have to be passed from the top down, from the access network to outgoing link (refer Fig. 3), while data have to be processed in the reverse direction at destinations. Equations (1) to (4) are used to calculate the nodal power consumption along with the values in Tables I and II. Equation (1) is the power consumption of the IP layer, and Equation (2) describes the OEO power consumption. $L_{I N}$, $L_{D}$, and $A_{O U T}$ are assumed equal to zero at sources, whereas $A_{I N}, L_{A}$, and $L_{O U T}$ are zero at destinations. Since only source and destination nodes involve OEO conversion, and so Equations (1) and (2) are not applied at any intermediate nodes along an all optical route. Equation (3) considers the consumed electricity in optical communication, which is calculated at all involved nodes. $\alpha$ is equal to $0.085 \mathrm{~kW}$ and represents the power consumption of amplifiers built into the node. The $\beta$ value is constant for directing the lightpaths to the appropriate ports and equals $0.15 \mathrm{~kW}$. The total power consumption is then calculated by summation of power consumption at all layers of the node using Equation (4).

\section{Least Cost and Least Emission Path Routing}

The authors present initial findings on reduction of electricity cost in unicast [13], and anycast [14]. We have perviously shown that Least Dollar Path (LDP) in the anycast case could lead to $7 \%$ improvement over standard routing approaches in average [13]. In this paper, we extend our anycast model to Least Emission Path (LEP). Our goal is minimizing the electricity cost and emissions to transport data. We also propose an analytical model to calculate the electricity cost and emissions for anycast connection.
LEP is a dynamic routing calculation technique. The dynamic routing calculation changes the weight of each link every $t$ units of time [15]. Therefore, the routes might also dynamically change at every interval of $t$. LEP recomputes the routes based on the real-time emissions factor, and $t$ is the known interval of updating emissions factor among all power markets. Electricity is consumed only at nodes in networks. Emissions of a path is proportional to emissions factor in destination nodes of each link of the path. Thus link weights are assigned according to emissions at destination nodes. Equation (5) describes the link weight assignment.

$$
W_{(i, j)}=\Gamma_{j} .
$$

Where $W_{(i, j)}$ is the weight of link $(i, j) . \Gamma_{j}$ is calculated from Equation (4) by:

$$
\Gamma_{j}=\gamma_{j} \times P_{j} .
$$

Where $\gamma_{j}$ is the emissions factor at node $j$.

The total electricity emissions of the path between node $s$ and node $d$ is calculated by:

$$
\Gamma_{(s, d)}=\sum_{n \in p_{s d}}^{N} \Gamma_{n} .
$$

Where $p_{s d}$ is the physical path between node $s$ and node $d$, and $\Gamma_{n}$ is calculated from Equation (4).

We assume routing and provisioning for Immediate Reservation (IR) lightpath requests, which has a specified bandwidth and duration. The data transmission required to be transmitted immediately upon the request arrival, otherwise will be blocked. Each request consists of a set of locations that hold the relevant replicas and a user-node for the data. The goal of the proposed algorithm is to pick the most green route and replica combination to incur the minimum emissions. We compare the proposed algorithm with three routing approaches Least Dollar Path (LDP), Shortest Hop Path (SHP) which finds routes with minimum number of intermediate nodes between user and any replica, and Shortest Distance Paths (SDP) which can find the path with minimal fiber distance between user and any replica.

Algorithm 1 shows the LEP algorithm, where $L_{r}$ is the replicas set, $u$ is the user, and $\tau$ denotes the time of service.

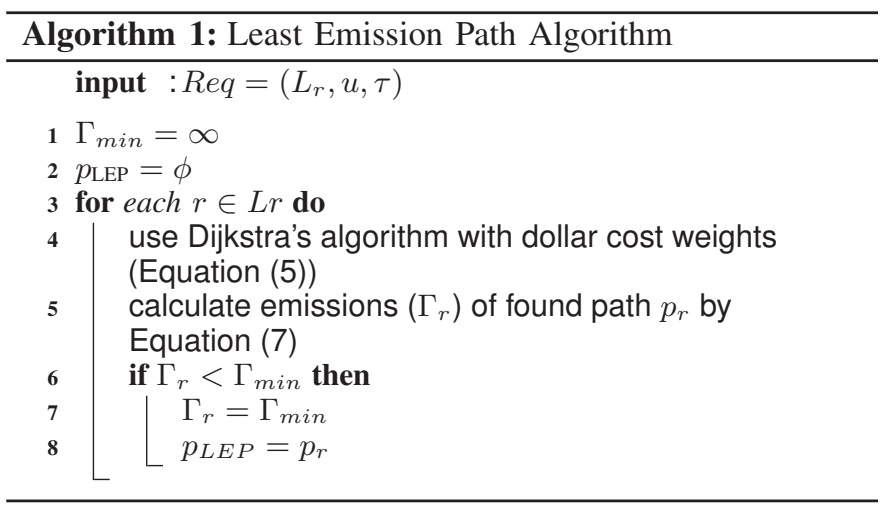


We proposed a similar algorithm for LDP in our previous work [14]. The complexity of Dijkstra's algorithm is $O(E+$ $N * \log (N))$ worst-case runtime, where the $E$ denotes the number of edges in the network, and $N$ represents number of nodes. The worst time complexity of LDP is $O(r)$, where $r$ is the number of replicas. Therefore, the total time complexity is $O(r(E+N * \log (N))+N)$.

\section{Analytical Modeling}

We now present the analytical modeling for calculating electricity cost in the core networks. We denote three kind of traffic for each node, in order to electricity cost and emissions anycast modeling: First the $\lambda_{\text {ns }}$ is the arrival rate of node $n$ denoting the traffic that starts from node $n$. Second, $\lambda_{n d}$ is the arrival rate of node $n$ for the traffic that has ended at the node $n$. Third, we denote the traffic that is passing the node $n$ as intermediate node as $\lambda_{\text {ni }}$. Due to using the Poisson process for traffic arriving and exponential distribution of time of service, the $\lambda_{\mathrm{ns}}$ is:

$$
\lambda_{n s}=\frac{\lambda_{\mathrm{t}}}{N}
$$

where $\lambda_{\mathrm{t}}$ is the arrival rate of entire network.

In anycast connection with $n_{\mathrm{d}}$ number of replicas, $\lambda_{\text {nd }}$ and $\lambda_{\mathrm{ni}}$ are dependent on the routing algorithms. Thus, $\lambda_{\text {nd }}$ and $\lambda_{\text {ni }}$ may be calculated as

$$
\begin{aligned}
\lambda_{n i} & =\frac{U_{\mathrm{n}}}{N\left(\begin{array}{c}
N-1 \\
n_{\mathrm{d}}
\end{array}\right)} \times \lambda_{\mathrm{t}} \\
\lambda_{n d} & =\frac{D_{\mathrm{n}}}{N\left(\begin{array}{c}
N-1 \\
n_{\mathrm{d}}
\end{array}\right)} \times \lambda_{\mathrm{t}}
\end{aligned}
$$

where $U_{\mathrm{n}}$ is the number of paths which use node $n$ as intermediate node, and $D_{\mathrm{n}}$ denotes number of paths which has ended to node $n$, out of all source and destination set combinations. $N\left(\begin{array}{c}N-1 \\ n_{\mathrm{d}}\end{array}\right)$ represents all possible combinations of source and destination sets.

Due to Section III the power consumption in source, destination, and intermediate nodes are:

$$
P_{\mathrm{n}}=\left(\lambda_{n s}+\lambda_{n d}\right)\left(p_{\mathrm{WDM}}+p_{\mathrm{OEO}}+p_{\mathrm{IP}}\right)+\lambda_{n i}\left(p_{\mathrm{WDM}}\right) .
$$

Thus, the total power consumption is calculated as

$$
\begin{gathered}
P=\sum_{n=1}^{N}\left(\lambda_{n s}+\lambda_{n d}\right)\left(p_{\mathrm{WDM}}+p_{\mathrm{OEO}}+p_{\mathrm{ip}}\right) \\
+\lambda_{n i}\left(p_{\mathrm{WDM}}\right)
\end{gathered}
$$

Due to Section II, the arrival rate fluctuate in oder to change the customer usage or routing algorithms priorities. Thus, we chunk the time to a shorter period that all above values are constant. So, the total power consumption is:

$$
\begin{gathered}
P=\sum_{\tau=1}^{T} \sum_{n=1}^{N}\left(\lambda_{n s \tau}+\lambda_{n d \tau}\right)\left(p_{\mathrm{WDM}}+p_{\mathrm{OEO}}+p_{\mathrm{ip}}\right) \\
+\lambda_{n i \tau}\left(p_{\mathrm{WDM}}\right)
\end{gathered}
$$

Due to using the exponential distribution for time of service, the total energy consumption is calculated by multiplexing the $\mu$ to the power consumption of network.

$$
\begin{gathered}
E=\sum_{\tau=1}^{T} \sum_{n=1}^{N} \mu\left\{( \lambda _ { n s \tau } + \lambda _ { n d \tau } ) \left(p_{\text {WDM }}+\right.\right. \\
\left.\left.p_{\text {OEO }}+p_{\text {ip }}\right)+\left(\lambda_{n i \tau}\right)\left(p_{\text {WDM }}\right)\right\}
\end{gathered}
$$

The total electricity cost of network may be calculated as

$$
\begin{gathered}
C=\sum_{\tau=1}^{T} \sum_{n=1}^{N} \mu\left\{( \lambda _ { n s \tau } + \lambda _ { n d \tau } ) \left(p_{\mathrm{WDM}}+\right.\right. \\
\left.\left.p_{\text {OEO }}+p_{\text {ip }}\right)+\left(\lambda_{n i \tau}\right)\left(p_{\mathrm{WDM}}\right)\right\} E P_{n \tau}
\end{gathered}
$$

Where $E P_{n \tau}$ is the real-time electricity price in node $n$ at period $\tau$. In the similar manner, total emissions is

$$
\begin{gathered}
\Gamma=\sum_{\tau=1}^{T} \sum_{n=1}^{N} t_{\text {service }}\left\{( \lambda _ { n s \tau } + \lambda _ { n d \tau } ) \left(p_{\text {WDM }}+\right.\right. \\
\left.\left.p_{\text {OEO }}+p_{\text {ip }}\right)+\left(\lambda_{n i \tau}\right)\left(p_{\mathrm{WDM}}\right)\right\} \gamma_{n \tau}
\end{gathered}
$$

Where $\gamma_{n \tau}$ is the real-time emissions factor of node $n$ at period $\tau$.

\section{Vi. Performance Evaluation}

In this section, we first evaluate the analytical model with simulation results, and then compare four algorithms, to show the electricity cost and emissions improvements. we finally show optimal replica placements based on emissions and electricity cost for 24-node NSFnet (refer Fig. 2). We tried the analytical model on the 14-node NSFnet and 9-node ring as well but omit results due to space constraints.

Calculations were done for $10 \mathrm{Gbps}$, and 32 wavelengths. Time of service $\mu$ follows an exponential distributions, and equal to 1 minute.

\section{A. Comparison between simulation results and analytical results}

We now compare the simulation results and analytical results for 24-node NSFnet. We set the electricity prices and emissions factor to fixed measure, and then increase the traffic loads. Thus the total electricity price and emissions increase linearly, similar to increasing the traffic load.

Fig. 4 and Fig. 5 show total electricity cost and emissions for 24-node NSFnet. In this comparison, we present the comparison for 2 and 3 replicas. It is clear that the analytical model calculate accurate results. Due to maximum $0.05 \%$ differences between simulation and analytical results, we use the analytical models in the remainder of the evaluation section.

Fig. 4 and Fig. 5 also show in increase the number of replica leads to decrease of total electricity cost and emissions. Considering the cost of maintaining more replicas and keeping them synchronized, Microsoft use three replicas for any actions [4]. Thus, we investigate the numerical result with three replicas in next subsections. 


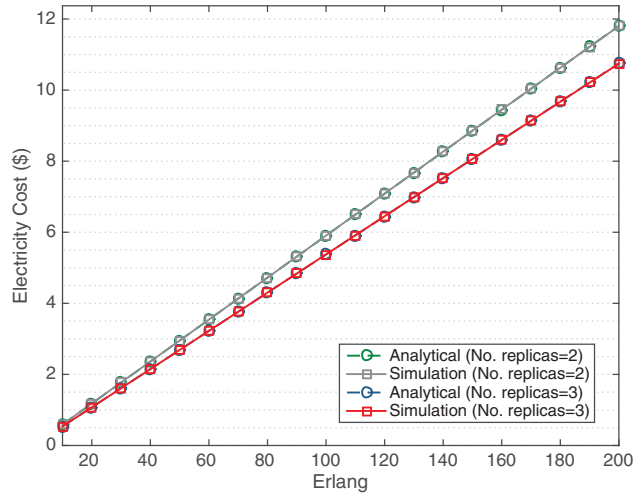

Fig. 4: Comparison Electricity Cost by Analytical Model and Simulation Model.

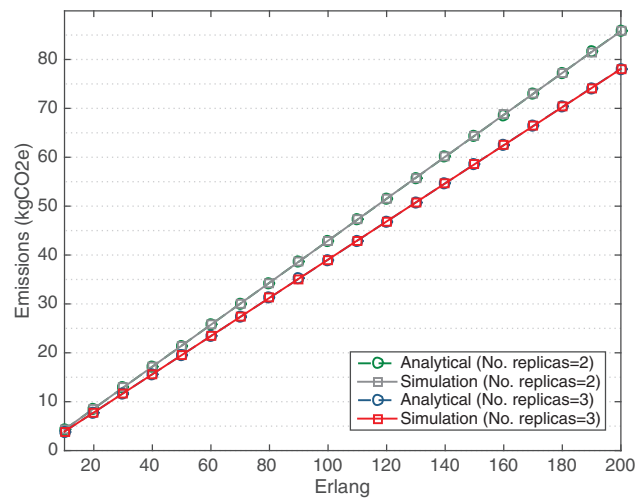

Fig. 5: Comparison Total Emissions by Analytical Model and Simulation Model.

\section{B. $L E P, L D P, S H P$, and $S D P$}

In this subsection, we first explain our simulation assumptions and different price and emissions factor environments. We evaluate the LDP approach on the 14-Node NSFnet topology in Fig. 2. Due to Fig. 2, Nodes are distributed across the country in four different time zones and thus will experience different peak hours; East-coast time is used as a reference in our simulations. Moreover, network nodes are classified in two categories: nodes are located in the one power markets, that have access to real-time electricity price and emissions factor. In the second nodal categories, there are not available electricity markets, therefor they have only access to flat electricity price and emissions factor. In this paper, we assume all nodes that have RTP available use it as their electricity charge function. For the flat rate nodes, we use the USA Energy Information Administration (EIA) average commercial rates for each node ${ }^{5}$. For the remaining nodes, we generate prices proportionally based on actual RTP compiled by ComEd Inc. ${ }^{6}$ for first week of June 2014 and also July 2014. We compare results of LED and LDP with the two baseline algorithms; SHP and SDP. We evaluate our results in terms of electricity cost improvement and emissions improvement. We study dynamic traffic arriving in a Poisson process with exponential holding times. The arrival rate is changing throughout the day as shown in Fig. 6.

\footnotetext{
${ }^{5} \mathrm{http}: / /$ apps1.eere.energy.gov/sled/

${ }^{6}$ https://rrtp.comed.com/live-prices/?date $=20140713$
}

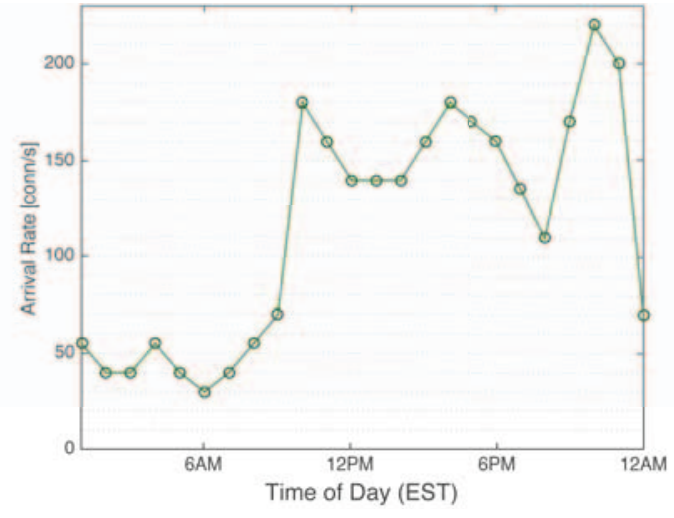

Fig. 6: Distribution of arrival rates throughout the day [16].

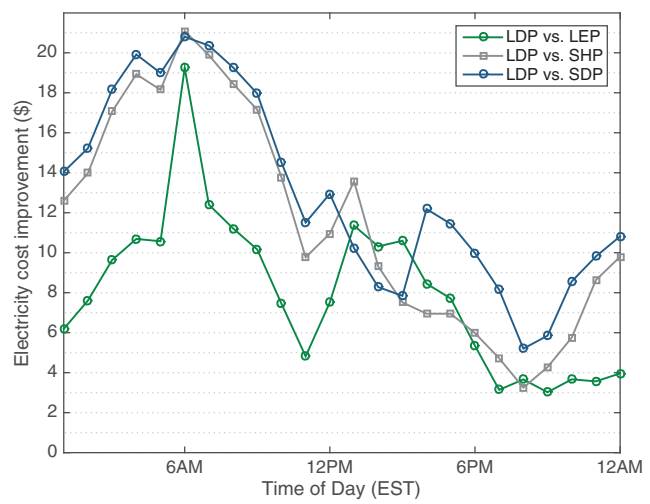

Fig. 7: Average cost improvement.

All candidate replicas and destinations are chosen randomly according to a uniform distribution [4].

Figure 7 shows the electricity cost improvement results in LEP, SHP, and SDP against LDP over a 24 hour period. Increasing the electricity price difference among nodes leads to huge electricity cost improvements. This scenario happens at midnight when the electricity prices in nodes that are charged with flat rate are still high. The difference of the electricity prices between nodes with and without RTP are much higher during the night than during the daytime. The night hours improvement is caused by the different kinds of electricity contracts. A higher number of nodes that are not covered by any power markets lead to these amounts of improvements.

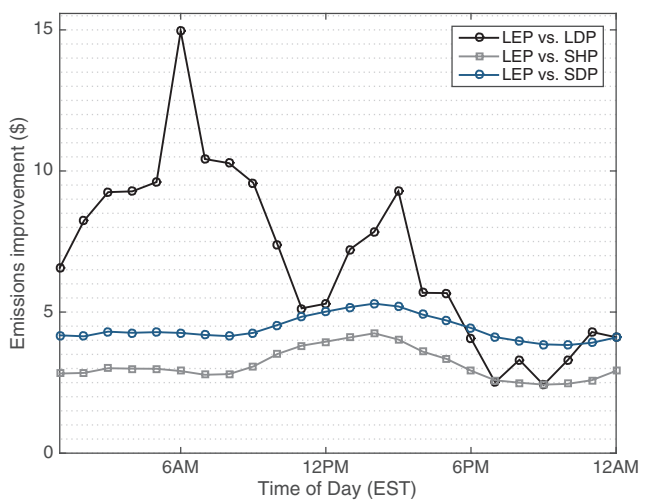

Fig. 8: Average emissions improvement. 
In Fig. 8 we plot the emissions reductions of LDP over LEP, SHP and SDP for three replicas over a 24 hour period. Reductions are between 3 and $4 \%$ due to the geographical distribution of the network, different time zones and also different markets price effect. The LDP, SHP and SDP are not aware of the gap between emissions for some nodes at noon, when the emissions factors are dropped in the nodes with photovoltaic power plants. This improvement can be even higher in some conditions such as abnormally wind speed at one node, when the emissions factors is three or four times more than normal emissions factor. Although rare, these events occur and LEP offers protection against the excessive charges

\section{Optimal Replica Placement}

Table III shows the best and worst combinations of replicas for four algorithms. We investigate all 2024 possible combinations of three replicas in network with 24 nodes, and have sorted data based on different parameters. We have included the worst combination to emphasize the range of value, in any case. All algorithm process are the same as the previous section; except that choosing replicas is not random. We defined a specific combination of three replicas for all requests. The table shows if three replicas have been selected based on LEP they have better emissions result in comparison with LDP, SHP and SDP. While the electricity cost in LDP improved more than $2 \%$ in comparison with LEP. The combination of node number 3 (California), 13 (Texas), and one of 11 (Illinois) or 16 (Kentucky) are the most interesting combination in terms of emissions and electricity cost. They consume lower electricity price and we also located in middle of country (refer Fig. 2). All the best combinations in electricity cost and emissions show that nodes with lower electricity price and emissions factor in middle of country could be ideal locations for replica.

\section{CONCLUSION}

In this paper we discuss how we can use real-time electricity prices and emissions factors to reduce power expenses and pollutions. We investigate energy aware routing for replicated content end nodes. Numerical results shows that the LDP and LEP approach is able to reduce electricity cost and emissions up to 22 percent in comparison to other algorithms. This amount of improvement incentivizes the implementation of those new routing methods and the use of devices with proportional energy use.

TABLE III

Optimal Placement of Three Replicas Using Algorithms: LDP, LEP, SHP, and SDP.

\begin{tabular}{|c|c|c|c|c|c|}
\hline & \multicolumn{2}{|c|}{ Emissions (kg) } & \multicolumn{2}{|c|}{ Electricity Cost $(\$)$} \\
\hline & & Replicas & Value & Replicas & Value \\
\hline \multirow{2}{*}{ LDP } & Best & {$[3,13,15]$} & 1591.6 & {$[3,13,11]$} & 242.3 \\
\hline & Worst & {$[1,2,4]$} & 1709.5 & {$[4,5,8]$} & 260.96 \\
\hline \multirow{2}{*}{ LEP } & Best & {$[3,13,16]$} & 1586.0 & {$[3,11,14]$} & 246.9 \\
\hline & Worst & {$[18,23,24]$} & 1683.1 & {$[4,5,8]$} & 264.77 \\
\hline \multirow{2}{*}{ SHP } & Best & {$[3,14,16]$} & 1587.0 & {$[3,11,14]$} & 246.6 \\
\hline & Worst & {$[1,2,4]$} & 1705.1 & {$[4,5,8]$} & 262.50 \\
\hline \multirow{2}{*}{ SDP } & Best & {$[5,14,16]$} & 1589.9 & {$[3,11,14]$} & 247.7 \\
\hline & Worst & {$[1,2,4]$} & 1696.7 & {$[1,2,4]$} & 262.15 \\
\hline
\end{tabular}

\section{REFERENCES}

[1] G. Smaragdakis, N. Laoutaris, K. Oikonomou, I. Stavrakakis, and A. Bestavros, "Distributed server migration for scalable internet service deployment," IEEE/ACM Transactions on Networking (TON), vol. 22, no. 3, pp. 917-930, 2014

[2] S. Agarwal, J. Dunagan, N. Jain, S. Saroiu, A. Wolman, and H. Bhogan, "Volley: Automated data placement for geo-distributed cloud services." in NSDI, 2010, pp. 17-32.

[3] A. Qureshi, "Power-demand routing in massive geo-distributed systems," Ph.D. dissertation, Massachusetts Institute of Technology, 2010.

[4] C. Huang, H. Simitci, Y. Xu, A. Ogus, B. Calder, P. Gopalan, J. Li, $\mathrm{S}$. Yekhanin et al., "Erasure coding in windows azure storage." in USENIX Annual Technical Conference. Boston, MA, 2012, pp. 15-26.

[5] J. M. Plante, D. A. Davis, and V. M. Vokkarane, "Parallel circuit provisioning in ESnet's OSCARS," in Advanced Networks and Telecommuncations Systems (ANTS), 2014 IEEE International Conference on, Dec 2014, pp. $1-6$.

[6] H. Zang, J. P. Jue, B. Mukherjee et al., "A review of routing and wavelength assignment approaches for wavelength-routed optical WDM networks," Optical Networks Magazine, vol. 1, no. 1, pp. 47-60, 2000.

[7] D.-R. Din, "A hybrid method for solving ARWA problem on WDM network," Computer Communications, vol. 30, no. 2, pp. 385-395, 2007

[8] T. Stevens, M. De Leenheer, C. Develder, F. De Turck, B. Dhoedt, and P. Demeester, "Anycast routing algorithms for effective job scheduling in optical grids," in Proceedings of ECOC 2006, the 32nd European Conference on Optical Communication, 2006, pp. 371-372.

[9] M. Mills, "The cloud begins with coal: Big data, big networks, big infrastructure, and big power," An Overview of the Electricity Used by the Global Digital Ecosystem. National Mining Association and American Coalition for Clean Coal Electricity, 2013.

[10] [Online]. Available: http://www.eia.gov/tools/faqs/faq.cfm?id=427\&t=3

[11] H. Allcott, "Rethinking real-time electricity pricing," Resource and Energy Economics, vol. 33, no. 4, pp. 820-842, 2011.

[12] W. Van Heddeghem, F. Idzikowski, W. Vereecken, D. Colle, M. Pickavet, and P. Demeester, "Power consumption modeling in optical multilayer networks," Photonic Network Communications, vol. 24, no. 2, pp. 86-102, 2012.

[13] A. Deylamsalehi, T. Schondienst, and V. M. Vokkarane, "Real-time energy price aware network routing," in High-capacity Optical Networks and Emerging/Enabling Technologies (HONET), 2014 11th Annual. IEEE, 2014, pp. $15-19$

[14] A. Deylamsalehi, P. Afsharlar, and V. M. Vokkarane, "Real-time energy price-aware network anycast rwa in optical data center network," in Computing, Networking and Communications (ICNC), 2016 International Conference on, 2016.

[15] G. P. Thodime, V. M. Vokkarane, and J. P. Jue, "Dynamic congestionbased load balanced routing in optical burst-switched networks," in Global Telecommunications Conference, 2003. GLOBECOM'03. IEEE, vol. 5. IEEE, 2003, pp. 2628-2632.

[16] M. Gattulli, M. Tornatore, R. Fiandra, and A. Pattavina, "Low-carbon routing algorithms for cloud computing services in ip-over-wdm networks," in Communications (ICC), 2012 IEEE International Conference on, 2012, pp. 2999-3003. 